\title{
CHILDHOOD HEARING LOSS AND RISK PROFILE IN A SOUTH AFRICAN POPULATION
}

De Wet Swanepoel ${ }^{1,2,3}$

Lorné Johl ${ }^{1}$

Danelle Pienaar ${ }^{1}$

1. Department of Communication Pathology, University of Pretoria, South Africa

2. Ear Sciences Centre, School of Surgery, the University of Western Australia, Nedlands, Australia

3. Ear Science Institute Australia, Subiaco, Australia

\section{Corresponding author:}

Prof. De Wet Swanepoel

Department of Communication Pathology

University of Pretoria

C/o Lynnwood \& University Roads

Hatfield, 0002

South Africa

Dewet.swanepoel@up.ac.za 


\section{ABSTRACT}

Objective: To describe the nature of hearing loss and associated risk profile in a representative South African population of infants and children diagnosed at a pediatric referral clinic.

Methods: A retrospective review of patient files for a pediatric auditory evoked potential clinic in Pretoria was conducted (January 2007 to December 2011).

Collected data included demographical information, risk factors from case history questionnaire, diagnosis (type and degree of hearing loss), documented age of caregiver suspicion and age of first diagnosis.

Results: Hearing loss was present in 73\% (73/100) of cases evaluated. Permanent hearing losses (SNHL, ANSD \& Mixed) constituted 76\% of losses. Unilateral hearing losses constituted $8 \%$ of SNHL and $20 \%$ of conductive hearing loss. ANSD was diagnosed in $21.4 \%$ and SNHL in $78.6 \%$ of permanent non-conductive hearing loss cases. The most prevalent SNHL risk was family history of hearing loss and for ANSD it was admittance to the NICU for more than 5 days. The majority of the sample was diagnosed with a permanent bilateral SNHL and ANSD after 36 months of age $(47 \%)$ despite $40 \%$ already suspected of having a hearing loss before 12 months of age.

Conclusions: A high prevalence of ANSD was found with preventable risk factors often indicated. Age of diagnosis was significantly delayed, evidencing the lack of early hearing detection services in South Africa. The majority of children were diagnosed at ages precluding optimal benefits from early detection and subsequent intervention. 


\title{
Keywords
}

Auditory neuropathy; hearing loss; infant; children; hyperbilirubinemia, risk factors; sensorineural, conductive.

\begin{abstract}
Abbreviations
ANSD - Auditory neuropathy spectrum disorder

PCEHL - Permanent congenital and early-onset hearing loss

SNHL - Sensorineural hearing loss
\end{abstract}

\section{INTRODUCTION}

More than 1 million babies are born annually in South Africa (1 059417 in 2011) of which very few will be afforded the opportunity to have their hearing screened $[1,2,3]$. Despite the proven benefits of early detection of hearing loss and early intervention it is still uncommon practice in South Africa [3]. A survey of early detection services in the public health care system, which serves approximately $85 \%$ of the South African population [4], indicate that less than $7.5 \%$ of hospitals offer any infant hearing screening services [5]. The private health care system provides slightly better coverage with $53 \%$ of obstetric units offering some form of screening but only $14 \%$ offering universal newborn hearing screening [2]. Existing programs are also not sufficiently systematic and are plagued by suboptimal and variable protocols for early detection, follow-up and data management [2]. As a result it is estimated that less than $10 \%$ of South African newborns are likely to have their hearing screened $[2,5]$. 
Unsurprisingly the average age of hearing loss diagnosis has been reported to be between 23 to 31 months of age as opposed to the recommended 3 months of age $[3,6,7]$.

Due to limited newborn hearing screening programs, resultant late identification, and insufficient data management $[2,3,5]$ there is very limited systematic data on the nature and causes of permanent congenital and early onset hearing loss (PCEHL) in South Africa. The only reports date back to the 1970's and early 80 's when a series of retrospective reviews of children in schools for the deaf were conducted across the country [8]. Within the diverse sample of 3064 school-aged children $25 \%$ presented with an acquired hearing loss, $7 \%$ with syndromic hearing loss, $11 \%$ with non-syndromic (familial) hearing loss and $57 \%$ with unknown causes $(11 \%$ with other anomalies and $46 \%$ without other anomalies). The main risks associated with acquired deafness were maternal rubella, meningoencephalitis, "severe illness", jaundice, birth trauma and prematurity [8]. Since these results were reported much has changed in terms of the specification of risk factors and even the diagnostic categories of hearing loss (i.e. Auditory Neuropathy Spectrum Disorder (ANSD)).

Recent reports have highlighted that developing regions such as sub-Saharan Africa may have risk factors for PCEHL that vary significantly from those established for developed world regions [9] and subsequently may present with a larger incidence of ANSD [10]. Unique developing world risk factors such as undernutrition, maternal high blood pressure and unskilled birth attendants has been associated with congenital and early-onset hearing loss in Nigeria $[9,11]$. Alongside such unique risk factors is a higher incidence of existing risk factors (e.g. birth trauma, asphyxia, 
neonatal jaundice, ototoxicity) associated with poor maternal and child health services typical of many developing world regions $[10,11,13,14]$. The prevalence of ANSD has been reported to be as high as $16 \%$ in a population of Nigerian children with sensorineural hearing loss (SNHL) born outside hospital as opposed to a prevalence of $10 \%$ in a similar group born within hospital [10].

South Africa is characterized by diversity in culture, language and economic development, being classified as an upper middle-income country, largely developing with pockets of developed contexts [15]. To date there has been no description of the nature of PCEHL in South Africa. Apart from studies conducted in deaf schools three to four decades ago [8], no associated context-specific risk factors have been documented for childhood hearing loss. The current study therefore describes the nature of hearing loss and associated risk profiles in a South African population of infants and children diagnosed with hearing loss at a referral clinic.

\section{METHODS}

Approval from the institutional ethics committee was obtained before any data collection commenced.

\section{Study Population}

A retrospective review of patient files for the pediatric auditory evoked potential clinic at the University of Pretoria was conducted from January 2007 to December 2011. The clinic is scheduled once a week during university terms serving as a referral source in Pretoria and surrounding areas. Few public healthcare hospitals offer this 
type of diagnostic service in Pretoria and surrounding areas. If services are available waiting lists typically exceed 6 months. Evaluations were conducted by experienced pediatric audiologists employed at the University of Pretoria with support from final year audiology students. Test batteries comprise mostly objective test procedures including acoustic immittance measurements, otoacoustic emissions, auditory brainstem responses, auditory steady-state responses supplemented by behavioral audiometric procedures where possible.

\section{Procedures}

Records of all the patients who attended the pediatric auditory evoked potential clinic between January 2007 and December 2011 were reviewed in order to locate and obtain the patient files kept in the Department of Communication Pathology at the University of Pretoria. Files were drawn from the filing cabinet and relevant information documented onto a data collection sheet developed to assist the researchers in sorting, analyzing and organizing the data. Data collected included demographical information, risk factors documented on the case history questionnaire, test procedures conducted, diagnosis (type and degree of hearing loss), documented age of caregiver suspicion and age of first diagnosis. The captured data was subsequently transferred from the data collection sheet to an electronic database.

\section{Data analysis}

Data was analyzed on a statistical software package (IBM SPSS version 19).

Descriptive measures were employed to describe the central tendency and normal distribution of recorded variables. A non-parametric test, the Mann-Whitney test, was 
employed to compare means between independent sub-samples using a significance level of $5 \%$.

\section{RESULTS}

Comprehensive diagnostic assessment information was obtained from the files of 100 children attending the paediatric hearing clinic at the University of Pretoria between January 2007 and November 2011. The rest of the files were incomplete and in some cases could not be sourced. More than half (53\%) of the patients' caregivers were first language speakers of an African language as opposed to English (12\%) and Afrikaans (35\%). Less than half (46\%) had access to some form of private medical aid. Referral sources for paediatric audiological assessments were from public health care hospitals $(52 \%)$, speech-language therapists $(14 \%)$, audiologists (11\%), schools (10\%), ENT specialists (4\%) and other sources $(9 \%)$.

\section{Childhood hearing loss}

Of the 100 children $73 \%$ (73/100) presented with hearing loss. Table 1 provides a description of the types of hearing losses across the sample. Permanent hearing losses (SNHL, ANSD \& Mixed) constituted $76 \%$ of hearing losses of which $4 \%$ included an additional conductive component (Mixed). One in four hearing losses was purely conductive $(23 \%)$ in nature. Except for one case $(n=1 / 17)$ of bilateral atresia all the other conductive losses $(n=16 / 17)$, including the mixed losses $(n=3)$, were due to middle ear effusion related to otitis media. Unilateral hearing losses constituted $8 \%$ of SNHL and $20 \%$ of conductive hearing loss. ANSD constituted 
Table 1. Distribution of hearing losses ( $n=73) . n=$ number of cases; Mixed hearing loss $=$ SNHL \& Conductive

\begin{tabular}{lcccccc}
\hline & \multicolumn{2}{c}{ Bilateral } & Unilateral & \multicolumn{2}{c}{$\begin{array}{c}\text { Uni- \& } \\
\text { Bilateral }\end{array}$} \\
& $\mathbf{n}$ & $\%$ & $\mathbf{n}$ & $\%$ & $\mathbf{n}$ & $\%$ \\
\hline Sensorineural hearing loss & 37 & $51 \%$ & 4 & $5 \%$ & 41 & $56 \%$ \\
Auditory neuropathy & 12 & $16 \%$ & - & - & 12 & $16 \%$ \\
Mixed hearing loss & 3 & $4 \%$ & - & - & 3 & $4 \%$ \\
Conductive hearing loss & 13 & $18 \%$ & 4 & $5 \%$ & 17 & $23 \%$ \\
\hline TOTAL & $\mathbf{6 5}$ & $\mathbf{8 9 \%}$ & $\mathbf{8}$ & $\mathbf{1 1 \%}$ & $\mathbf{7 3}$ & $\mathbf{1 0 0 \%}$ \\
\hline
\end{tabular}

$21.4 \%$ and SNHL (including mixed hearing losses) constituted $78.6 \%$ of permanent hearing losses (SNHL, ANSD \& Mixed).

As illustrated in figure 1 approximately $50 \%$ of SNHL were of a profound degree (including moderate to profound, severe to profound and profound). All mixed hearing losses $(n=6)$ presented as a profound hearing loss. In $42 \%(n=10 / 24)$ of ears diagnosed with ANSD the degree of hearing could not be specified. The remaining distribution of ears diagnosed with ANSD presented with a profound $(57 \% ; n=8 / 14)$, severe $(29 \% ; n=4 / 14)$ or mild $(14 \% ; n=2 / 14)$ degree of hearing loss. Two thirds of ears with conductive hearing loss had a mild degree of hearing loss $(70 \% ; n=21 / 30)$ with a small number presenting with moderate $(20 \% ; n=6 / 30)$ and severe $(7 \%$; $n=2 / 30)$ degrees of hearing loss ( $3 \%$ had no degree specified). 


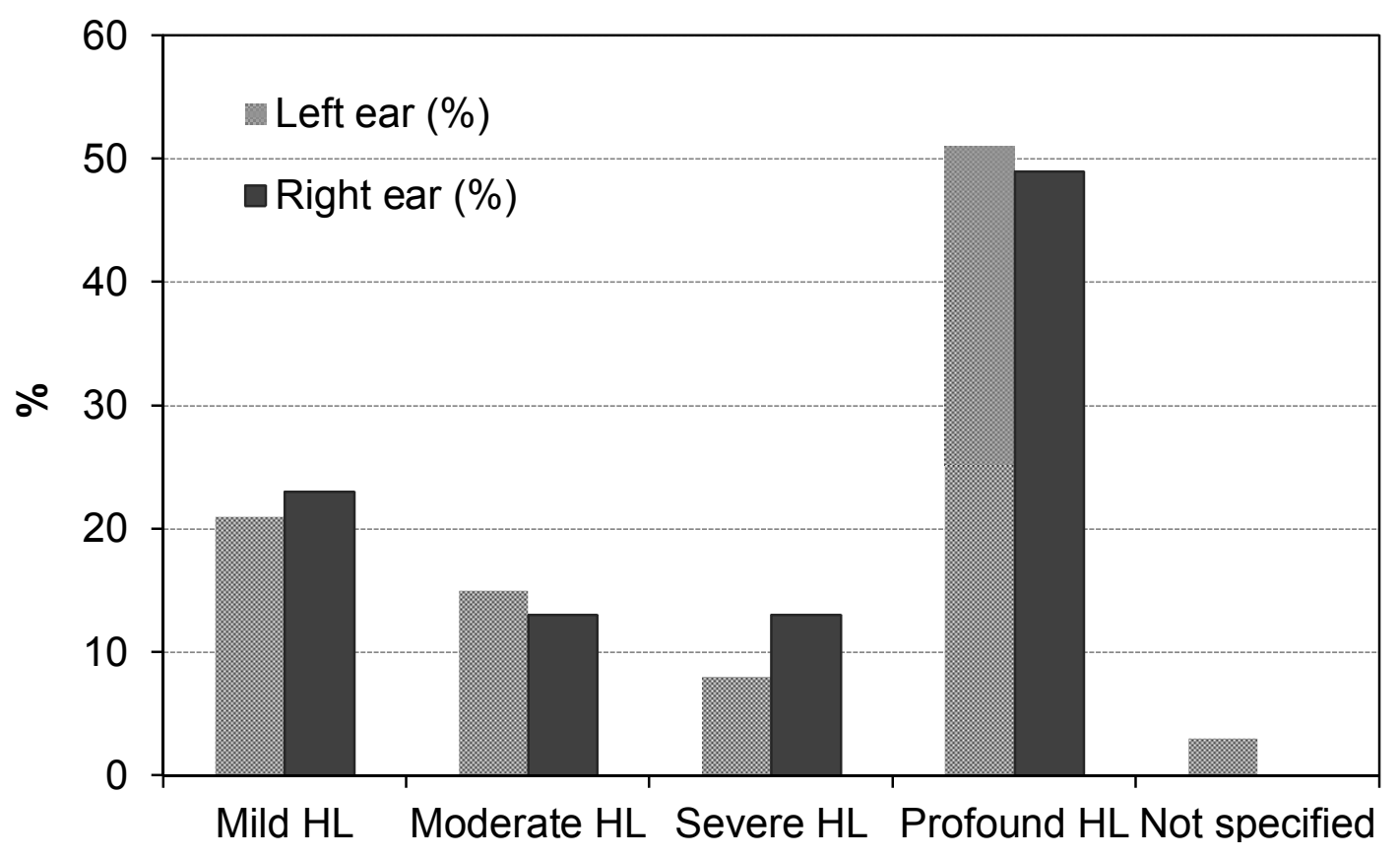

Figure 1. Distribution of sensorineural hearing loss degree (excluding ANSD) across ears $(n=78)$

\section{Risk profiles}

Table 2 provides a summary of the risk factors documented for the subjects with hearing loss. The syndromes included in the sample were Goldenhar, Cri-du-Chat and Prader Willi syndrome. The Cri-du-Chat subject was diagnosed with ANSD and the other with SNHL. The most prevalent SNHL risk was family history of hearing loss $(27 \%)$ and for ANSD it was admittance to the NICU for more than 5 days $(70 \%)$. 
Table 2. Documented risk factors ( $n=$ number of children affected with completed risk profiles)

\begin{tabular}{|c|c|c|c|c|}
\hline Risk Factors & $\begin{array}{l}\text { No HL } \\
(n=19) \\
\%(n)\end{array}$ & $\begin{array}{l}\text { SNHL } \\
(n=29) \\
\%(n)\end{array}$ & $\begin{array}{l}\text { ANSD } \\
(n=10) \\
\%(n)\end{array}$ & $\begin{array}{c}\text { SNHL \& } \\
\text { ANSD (n=39) } \\
\%(n)\end{array}$ \\
\hline Admittance to NICU > 5 days & $16(3)$ & $14(4)$ & $70(7)$ & $27.5(11)$ \\
\hline Hyperbilirubinemia & - & $3.5(1)$ & $50(5)$ & $15(6)$ \\
\hline Asphyxia & $10.5(2)$ & $10(3)$ & $50(5)$ & $20(8)$ \\
\hline Extremely LBW < 1500g & $21(4)$ & $3.5(1)$ & $40(4)$ & $12.5(5)$ \\
\hline Family history of hearing loss & $16(3)$ & $27.5(8)$ & $20(2)$ & $25(10)$ \\
\hline Syndrome present & $16(3)$ & - & $10(1)$ & $2.5(1)$ \\
\hline Congenital infections & - & - & $10(1)$ & $2.5(1)$ \\
\hline Craniofacial defects & - & $3.5(1)$ & - & $2.5(1)$ \\
\hline Bacterial meningitis & - & $3.5(1)$ & - & $2.5(1)$ \\
\hline
\end{tabular}

\section{Age of hearing loss suspicion and diagnosis}

Within the group diagnosed with bilateral permanent hearing loss (SNHL, ANSD \& Mixed hearing loss; $n=52$ ), only 36 caregivers reported the age at which they suspected the presence of a hearing loss for the first time (Table 3). The majority of the sample population was diagnosed with a permanent bilateral SNHL and ANSD after 36 months of age (47\%) despite $40 \%$ of this group already suspected of having a hearing loss before 12 months of age. A delay of 9 months or less between suspicion and diagnosis was evident for $25 \%$ of bilateral permanent losses. Only $20.4 \%$ of children with bilateral SNHL and ANSD were diagnosed before 18 months 
of age and less than one in three (30.6\%) before 24 months of age. Unilateral losses were suspected only after 36 months of age in $75 \%(n=3 / 4)$ of cases.

Table 3. Age of bilateral hearing loss suspicion and diagnosis $(n=$ number of individuals affected)

\begin{tabular}{lccc}
\hline & $\begin{array}{c}\text { Age at } \\
\text { suspicion } \\
\text { (months) }\end{array}$ & $\begin{array}{c}\text { Age at } \\
\text { diagnosis } \\
\text { (months) }\end{array}$ & $\begin{array}{c}\text { Delay from suspicion to } \\
\text { diagnosis (months) }\end{array}$ \\
\hline Mean & 23.7 & $n=49$ & $n=36$ \\
SD & 18.9 & 27.6 & 22.0 \\
Max & 72.0 & 128.2 & 16.3 \\
Min & 2.0 & 2.2 & 68.2 \\
& & & 0
\end{tabular}

There was no significant difference between the age of hearing loss suspicion and age of diagnosis for families with or without medical insurance $(p>0.05$; MannWhitney Test). No significant difference was also evident between the age of hearing loss suspicion for African first language compared to English and Afrikaans first language speaking families ( $p>0.05$; Mann-Whitney Test).

Average age of $\mathrm{HL}$ suspicion in cases of ANSD was 32 months (28.7 SD; 6 cases) compared to 22 months (16.2 SD; 30 cases) for bilateral SNHL but was not statistically significant however (Mann-Whitney; $p>0.05$ ). The average age of $\mathrm{HL}$ 
diagnosis in cases of ANSD was 45.1 months (41.1 SD; 12 cases) compared to bilateral SNHL of 41.1 months (22.6 SD; 37 cases) but was also not statistically significant (Mann-Whitney; $p>0.05$ ).

\section{DISCUSSION}

The cultural diversity and economic inequality of the South African population was represented in the research sample with the majority being African first language speakers $(53 \%)$, more than half $(54 \%)$ without private medical insurance and the majority $(52 \%)$ referred from public health care facilities $[1,4,15]$.

Three in every four children assessed at the clinic presented with a hearing loss of which $69 \%$ had a bilateral, and $7 \%$ had a unilateral permanent hearing loss (SNHL, ANSD \& mixed). ANSD cases constituted a larger proportion (21.4\%) of permanent hearing losses (SNHL, ANSD \& mixed) than previously reported. Previous reports have indicated prevalence for ANSD of between 5 to $17 \%$ of SNHL in children $[16,17,18,19]$. This prevalence has shown to increase when only severe to profound degrees of SNHL are considered [20]. The only other report from sub-Saharan Africa was from Nigeria, which reported ANSD prevalence of $15.9 \%$ in SNHL cases for a cohort of babies born outside of hospital and $10.3 \%$ for a cohort born in hospital [10]. The current study ANSD prevalence is still slightly higher and may be attributed in part to the nature of the research cohort, which comprised a group of children referred for diagnostic testing at a specialized university clinic. A complex case, typical of ANSD, is more likely to be referred for diagnosis at a specialized university clinic. Furthermore however it may also reflect the increased environmental and 
maternal and child health related risk factors (hyperbilirubinemia) predisposing ANSD in populations from developing contexts such as sub-Saharan Africa $[10,12]$.

Within the sample population diagnosed with ANSD the most prevalent associated risk factor was admittance to the NICU for longer than five days. Olusanya et al.,[21] indicated that more than half $(54.5 \%)$ of the children diagnosed with ANSD were admitted to hospital during the neonatal period for serious illness. NICU graduates have a significantly higher risk of ANSD as opposed to low-risk well baby populations $[7,22]$. Hyperbilirubinemia and asphyxia were the second most prevalent risk factors $(50 \%)$ associated with ANSD. This is in agreement with the multi-centre study by Berlin and colleagues [17] wherein $48 \%$ of ANSD cases presented with hyperbilirubinemia as risk factor. Hyperbilirubinemia is more prevalent in African countries due to a higher incidence of glucose-6-phosphate dehydrogenase deficiency and lack of adequate medical treatment $[13,23,24]$. The Berlin and colleagues [17] study also reported a high prevalence for asphyxia-related risks including anoxia (17\%), respiratory distress (15\%) and artificial ventilation (23\%).

The risks associated with SNHL were significantly less prevalent in comparison with the ANSD cases. The primary risk factor associated with SNHL was family history $(27.5 \%)$, which was significantly higher than the $11 \%$ previously reported for South African children with hearing loss [8]. There is the possibility of this risk factor being over-reported in the current study since the case history form, completed by caregivers, specifies "any family history of childhood hearing loss", which may include reports of transient conditions. Considering the increase in prevalence between those children with SNHL and those without, reveal the prevalence $(11.5 \%)$ 
to be similar to that of Sellars \& Beighton [8]. The other risks associated with SNHL included perinatal factors such as NICU admittance and asphyxia.

The degree of hearing loss was profound for $50 \%$ of SNHL ears and $57 \%$ of ANSD ears. A previous report on degree of hearing loss in a South African group of children indicated severe and profound degrees of hearing loss $(61 \%)$ similar to that of the current study [6]. In a multicentre study of ANSD cases, profound hearing loss was reported in $37 \%$ of ears [17].

There was a substantial delay between the age of hearing loss suspicion by caregivers and the age of eventual diagnosis in the study population. Caregivers generally suspected hearing loss by 23.7 months of age, although the average age of diagnosis of a permanent SNHL was only at 42.1 months indicating an average delay of 22 months. These long delays evidence the limited newborn hearing screening programs available in public and private health care sectors $[2,5]$. The result is passive detection relying on caregivers who usually suspect hearing loss only after critical language development milestones have already passed [3]. Average age of suspicion for ANSD (32 months) was later and more variable compared to SNHL (22 months). This may partly be related to the inconsistent auditory responses that often characterize this population [17].

The mean age of parental suspicion in a Nigerian sample of school-aged children was reported to be between 12 to 24 months of age with an average 18 month delay until diagnosis of a hearing loss [25]. Previous studies in South Africa of children surveyed in schools for the deaf $[6,26]$ reported average age of diagnosis to be 23 
months in the Western Cape and 31 months in the Gauteng provinces of South Africa[6,26]. The current study, also situated in the Gauteng province, presented an average age of diagnosis significantly later (42 months) than the previous reports. Most likely reasons for the difference between studies relate to the difference in sample populations. As opposed to an unselected population referred for diagnostic hearing assessment the previous report for Gauteng province [26] included a population of children already enrolled in early intervention programmes. As a result they are predisposed to be from more developed socio-economic settings with infant hearing screening being more likely and access to services being better $[2,3]$.

\section{CONCLUSION}

Hearing loss across this paediatric South African sample were typically permanent with a high prevalence of ANSD. Risks for ANSD were mostly preventable perinatal factors related to maternal and child health care. Age of hearing loss diagnosis was significantly delayed evidencing the lack of early detection services in the public and private health sectors of South Africa. The majority of children are diagnosed at ages which preclude optimal benefits from early detection and subsequent intervention.

\section{REFERENCES}

1. Statistics South Africa, 2011. Mid-year population estimates. http://www.statssa.gov.za/publications/P0302/P03022011.pdf. Accessed October 1, 2012. 
2. Meyer ME, Swanepoel D, Le Roux T, Van der Linde M. Early detection of infant hearing loss in the private health care sector of South Africa. Int $J$ Pediatr Otorhinolaryngol. 2012; 76(5): 698-703.

3. Swanepoel D, Storbeck C, Friedland P. Early hearing detection and intervention in South Africa. Int J Pediatr Otorhinolaryngol. 2009; 73(6): 783786.

4. Blecher M, Harrison S. Healthcare financing. In: Ijumba $P$, Padarath $A$, eds. South African Health Review 2006. Durban: Health Systems Trust, 2006. www.healthlink.org.za/uploads/files/chap3 06.pdf (accessed 1 June 2011).

5. Theunissen M, Swanepoel D. Early hearing detection and intervention services in the public health sector in South Africa. Int J Audiol. 2008; 47: 2329.

6. Van der Spuy T, Pottas L. Infant hearing loss in South Africa: Age of intervention and parental needs for support. Int J Audiol. 2008; 47: 30-35.

7. Joint Committee on Infant Hearing $(\mathrm{JCIH})$. Year 2007 position statement: Principles and guidelines for early hearing detection and intervention programs. Pediatrics, 2007; 120: 899-921.

8. Sellars S, Beighton P. Childhood deafness in Southern Africa: an aetiological survey of 3064 deaf children. Jf Laryngol Otol, 1983; 97: 885-9.

9. Olusanya BO. Neonatal hearing screening and intervention in resource-limited settings: an overview. Global Child Health. 2012. [Epub ahead of print]

10. Olusanya BO, Somefun AO. Sensorineural hearing loss in infants with neonatal jaundice in Lagos: a community-based study. Ann Trop Paediatr. 2009; 29(2):119-128. 
11. Olusanya BO. Making targeted screening for infant hearing loss an effective option in less developed countries. Int J Pediatr Otorhinolaryngol. 2011; 75: 316-321.

12. Swanepoel D. Judith Gravel Lecture,The Global Epidemic of Infant Hearing Loss-Priorities for Prevention. In: R.C. Seewald \& J.M. Bamford (eds.) A Sound Foundation Through Early Amplification: Proceedings of the Fifth International Conference, 2010:19-27.

13. Olusanya BO, Somefun AO. Place of birth and characteristics of infants with congenital and early-onset hearing loss in a developing country. Int $J$ Pediatr Otorhinolaryngol. 2009; 73(9):1263-1269.

14. Olusanya BO, Afe AJ, Solanke OA. Are risk factors for stillbirths in low-income countries associated with sensorineural hearing loss in survivors. J Matern Fetal Med. 2009; 22(7): 576-583.

15. World Bank, Africa Development Indicators 2007, World Bank Publications, Washington, DC, 2008.

16. Rance G, Beer DE, Cone-Wesson B. Clinical findings for a group of infants and young children with auditory neuropathy. Ear Hear. 1999; 20: 238-252.

17. Berlin Cl, Hood LJ, Morlet T, Wilensky D, Mattingly LLKR et al. Multi-site diagnosis and management of 260 patients with Auditory Neuropathy/Dyssynchrony (Auditory Neuropathy Spectrum Disorder). Int J Audiol. 2010; 49: $30-43$.

18. Ngo RY, Tan HK, Balakrishnan A, Lim SB, Lazaroo DT. Auditory neuropathy/auditory dys-synchrony detected by universal newborn hearing screening. Int J Pediatr Otorhinolaryngol. 2006; 70(7): 1299-306. 
19. Bielecki I, Horbulewicz A, Wolan T. Risk factors associated with hearing loss in infants: an analysis of 5282 referred neonates. Int J Pediatr Otorhinolaryngol. 2012; 75(7): 925-30.

20. Mittal R, Ramesh AV, Panwar SS, Nilkanthan A, Nair S, Mehra PR. Auditory neuropathy spectrum disorder: Its prevalence and audiological characteristics in an Indian tertiary care hospital. Int Pediatr Otorhinolaryngol. 2012; 76(9): 1351-1354. [Epub ahead of print].

21. Olusanya B, Wirz SL, Luxon LM. Factors associated with auditory neuropathy/dys-synchrony in a devloping country. Audiol Med. 2008; 6: 120128.

22. Korver AM, van Zanten GA, Meuwese-Jongejeugd A, van Straaten HL, Oudesluys-Murphy AM. Auditory neuropathy in a low-risk population: A review of the literature. Int J Pediatr Otorhinolaryngol. 2012. [Epub ahead of print]

23. Cappellini M, Fiorelli G. Glucose-6-phosphate dehydogenase deficiency. Lancet. 2008; 371(9606): 64-74.

24. Tarnow-Mordi WO, Pickering D. Missed jaundice in black infants: a hazard?. $\mathrm{Br}$ Med J (Clin Res Ed). 1983; 286(6363): 463-464.

25.Venter C, Viljoen J. Children with hearing loss: parental needs regarding diagnosis. Unpublished undergraduate research report, University of Pretoria, South Africa. 2008. 